Review

\title{
Self-Homodyne Detection in Optical Communication Systems
}

\author{
Benjamin J. Puttnam ${ }^{1}{ }^{1}$, Ruben S. Luís ${ }^{1}$, José Manuel Delgado Mendinueta ${ }^{1}$, Jun Sakaguchi ${ }^{1}$, \\ Werner Klaus ${ }^{1}$, Yukiyoshi Kamio ${ }^{1}$, Moriya Nakamura ${ }^{2}$, Naoya Wada ${ }^{1}$, Yoshinari Awaji ${ }^{1}$, \\ Atsushi Kanno ${ }^{1}$, Tetsuya Kawanishi ${ }^{1}$ and Tetsuya Miyazaki ${ }^{1}$
}

1 National Institute of Information and Communications Technology (NICT), 4-2-1 Nukui-kita, Koganei, Tokyo 184-8795, Japan; E-Mails: ruben.luis@nict.go.jp (R.S.L.); mendi@nict.go.jp (J.M.D.M.); jsakaguchi@nict.go.jp (J.S.); klaus@nict.go.jp (W.K.); kamio@nict.go.jp (Y.K.); wada@nict.go.jp (N.W.); yossy@nict.go.jp (Y.A.); kanno@nict.go.jp (A.K.); kawanish@nict.go.jp (T.K.); tmiyazaki@nict.go.jp (T.M.)

2 Department of Electronics and Bioinformatics, School of Science and Technology, Meiji University, 1-1-1 Higashimita, Tama-ku, Kawasaki 214-8571, Japan; E-Mail: m_naka@meiji.ac.jp

* Author to whom correspondence should be addressed; E-Mail: ben@nict.go.jp; Tel.: +81-42-327-5439; Fax: +81-42-327-7035.

Received: 2 March 2014; in revised form: 28 March 2014 / Accepted: 2 April 2014 / Published: 6 May 2014

\begin{abstract}
We review work on self-homodyne detection (SHD) for optical communication systems. SHD uses a transmitted pilot-tone (PT), originating from the transmitter laser, to exploit phase noise cancellation at a coherent receiver and to enable transmitter linewidth tolerance and potential energy savings. We give an overview of SHD performance, outlining the key contributors to the optical signal-to-noise ratio penalty compared to equivalent intradyne systems, and summarize the advantages, differences and similarities between schemes using polarization-division multiplexed PTs (PDM-SHD) and those using space-division multiplexed PTs (SDM-SHD). For PDM-SHD, we review the extensive work on the transmission of advanced modulation formats and techniques to minimize the trade-off with spectral efficiency, as well as recent work on digital SHD, where the SHD receiver is combined with an polarization-diversity ID front-end receiver to provide both polarization and modulation format alignment. We then focus on SDM-SHD systems, describing experimental results using multi-core fibers (MCFs) with up to 19 cores, including high capacity transmission with broad-linewidth lasers and experiments incorporating SDM-SHD in networking. Additionally, we discuss the requirement for polarization tracking of the PTs at the receiver and path length alignment and review some
\end{abstract}


variants of SHD before outlining the future challenges of self-homodyne optical transmission and gaps in current knowledge.

Keywords: self-homodyne coherent detection; coherent optical systems; multi-core fiber

\section{Introduction}

Self-homodyne detection (SHD) by using a transmitted pilot-tone (PT) as the local oscillator (LO) for coherent reception has long been proposed and investigated as a useful technique to minimize the impact of laser phase noise in optical communication systems [1-3]. The laser phase noise cancellation property of SHD systems arises from having the signal and LO originating from the same laser and preserving their coherence throughout the optical transmission path. This can relax requirements for narrow linewidth lasers [4-7], particularly when using phase-noise-sensitive, spectrally efficient, high-order modulation formats [8-10] or orthogonal frequency division multiplexing (OFDM) [11]. SHD may also reduce the complexity of the receiver and consequently provide energy savings by reducing the required digital signal processing (DSP) resources [12,13].

SHD using a PT polarization-division multiplexed to the data (PDM-SHD) has been demonstrated for a range of advanced modulation formats, including quaternary-amplitude modulation (QAM) with a constellation of 64 symbols [8,9] and OFDM [11]. However, one drawback of PDM-SHD is the trade-off of the spectral efficiency (SE) compared to polarization-division multiplexed modulation formats. Since it is only possible to modulate a single polarization with data signals, the SE of PDM-SHD systems can be reduced by up to 50\% compared to PDM transmission using intradyne detection (ID), although techniques, such as spectral interleaving of the PT and signal, may be used to improve the SE $[14,15]$. For this reason, SHD has recently been applied to space-division multiplexing (SDM) systems and specifically to multi-core fiber (MCF) technology [16-18]. SDM-SHD systems allow transmission of the PT through one dedicated SDM channel with the remainder used for signal-bearing channels. In this case, the SE reduction compared to an equivalent ID system becomes inversely proportional to the number of SDM channels and becomes comparable to the SE of ID systems for a high number of spatial channels. In SHD schemes, the path length alignment between signal and PT can impact phase noise cancellation, particularly in the presence of fiber non-linearities [3]. For SDM systems using MCF, it is envisaged that different cores of the same fiber experience the same environmental disturbances during transmission, and thus, path length variations between channels are minimized compared to a system comprising parallel single core or ribbon fibers [19].

In this paper, we review existing work on SHD and draw conclusions about the feasibility and gaps in current knowledge. In Section 2, we give an overview of SHD performance, outline the key contributors to the optical signal-to-noise ratio (OSNR) penalty and explain the advantages, differences and similarities between PDM-SHD and SDM-SHD. We also discuss some variants of SHD and the requirement for polarization tracking of the PT. In Section 3, we focus on PDM-SHD, reviewing the extensive work on using SHD to enable the transmission of advanced modulation formats, techniques to minimize the trade-off with spectral efficiency and recent work on digital SHD, where the SHD receiver is combined with an ID front-end receiver to provide both polarization and modulation format 
alignment. In Section 4, we focus on SDM systems and the differences with PDM-SHD. We review experimental results using MCF with up to 19 cores, including high capacity transmission using wide-linewidth lasers and experiments incorporating SHD in networking and discuss the requirement for path length alignment. Finally, in Section 5, we outline the future challenges of self-homodyne detection and research work required to make such systems attractive for commercial optical communication networking applications.

\section{Overview of Self-Homodyne Detection}

\subsection{Concept and Early Work}

The first experimental demonstration recognizable as SHD in optical fiber transmission was reported by Miyazaki and Kubota in 2005 in a development of homodyne coherent transmission systems, which, in the absence of sufficiently fast DSP, required optical phase locked loops [20] to lock the frequency and phase of the locally generated LO to the incoming signal. The original concept used a polarization multiplexed PT to demodulate $20 \mathrm{~Gb} / \mathrm{s}$ QPSK. As described in Section 3.1, the same approach was used for transmission demonstrations of high-order modulation formats with minimal modification before the advent of SDM and MCF technology led to the investigation of SHD with the PT transmitted on one core of a MCF. Schematics of both kinds of SHD schemes are shown in Figure 1.

Figure 1. Signal and pilot-tone transmission schemes: (a) polarization-division-multiplexed (PDM) and (b) space-division-multiplexed (SDM) self-homodyne detection (SHD) systems.

(a) PDM-SHD

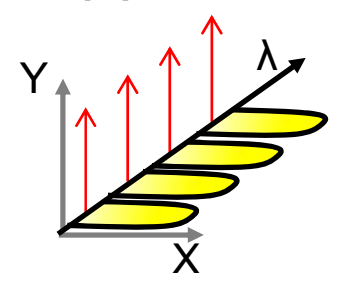

(b) SDM-SHD

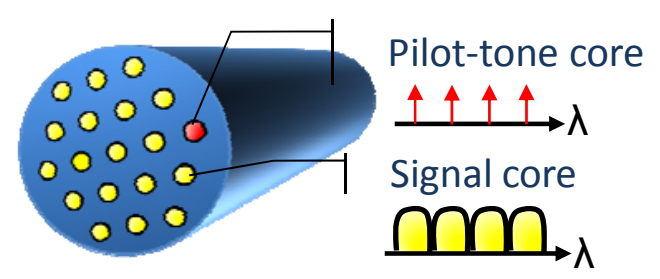

Although the implementation details of both schemes vary, the motivation, benefits and drawbacks of SHD are common. SHD offers the potential of removing the dependence of the laser linewidth on transmission performance by taking advantage of phase noise cancellation between the signal and LO that originate from the same laser. In addition, SHD reduces the receiver complexity both in terms of hardware and DSP resources by eliminating the LO from the coherent receiver, removing the need for frequency offset compensation and reducing the requirement for carrier phase recovery by several orders of magnitude, as discussed in Section 2.3. These benefits are traded off with the additional noise the PT accumulates during signal transmission and results in a receiver penalty, discussed in Section 2.2. Additionally, implementation difficulties arise, since it becomes necessary to maintain some correlation in the optical path lengths traversed by the interacting signal and PT (see Section 4.3), and effort must also be made to track or control the PT polarization at the receiver input, as discussed in Section 2.4. 


\subsection{Penalty Compared to Intradyne Detection}

In an SHD system, both the PT and signal accumulate amplified spontaneous emission (ASE) noise along the transmission path, resulting in a reduced post-detection, signal-to-noise ratio (SNR), compared to an equivalent ID system with similar signal optical SNR (OSNR) and a nearly noiseless, receiver-side generated LO [21]. The characteristics of noise after coherent detection in a SHD system differ from those of an ID system, due to additional contributions generated by the interactions of the PT and the signal noise, as well as the PT noise and signal noise. These yield beat noise contributions similarly to those observed in direct-detection systems [22]. In this subsection, we describe the performance of SDM-SHD systems to provide a general performance model, which is later adapted to the case of PDM-SHD systems.

To derive terms for the OSNR, consider the detection of a single or polarization-multiplexed signal and its corresponding PT at the input of a polarization-diverse coherent receiver. For simplicity, it is assumed that both the signal and PT polarization states are aligned with the optical axis of the receiver polarization beam splitter (PBS), which may in practice require an active control loop, as discussed in Section 2.4. The electrical current associated with the coherent detection of one of the signal polarization components may then be approximated by:

$$
i_{r}=2 \mathrm{R}\left(e_{s} e_{p}^{*}+n_{s} e_{p}^{*}+e_{s} n_{p}^{*}+n_{s} n_{p}^{*}\right) * h
$$

where $e_{s}$ and $e_{p}$ are the projections of the complex envelopes of the signal and PT fields over the analyzed polarization axis and the terms $n_{s}$ and $n_{p}$ are the corresponding ASE noise contributions of the signal and PT, respectively. $\mathrm{R}$ and $h$ are the responsivity of the photodetectors and the impulse response of the receiver front-end, respectively. The terms within parenthesis on the right-hand side of Equation (1) correspond to the detected signal, the signal ASE-PT beat noise, the signal-PT ASE beat noise and the signal ASE-PT ASE beat noise, respectively. Equation (1) assumes that ASE noise dominates the system performance, disregarding other noise sources, like thermal or shot noise. As an approximation, it will be assumed that the terms in Equation (1) are statistically independent, in which case, the variance of the electrical current conditioned to the detection of a given symbol with nominal power, $P_{i}$, may be approximated by:

$$
\sigma_{i}^{2}=\sigma_{p-n s}^{2}+\sigma_{s-n p, i}^{2}+\sigma_{n s-n p}^{2}
$$

The terms on the right-hand side of Equation (2) are the variances of the signal ASE-PT beat noise, the signal-PT ASE beat noise and the signal ASE-PT ASE beat noise, respectively. Note that in ID systems, only the term, $\sigma_{p-n s}^{2}$, is present and may be approximated by [23]:

$$
\sigma_{p-n s}^{2}=4 \mathrm{R}^{2} R_{s} N_{s} P_{p}
$$

where $R_{s}, N_{s}$ and $P_{p}$ are the signal symbol rate, the power spectral density (PSD) of the signal ASE and the average PT power, respectively. The signal dependent term, $\sigma_{s-n p, I}^{2}$, may be estimated assuming negligible inter-symbol interference, as:

$$
\sigma_{s-n p, i}^{2}=2 \mathrm{R}^{2} W_{p} N_{p} P_{i} / p
$$

where $W_{p}, N_{p}$ are the full-width at half maximum of the PT filter and the PSD of the PT ASE, respectively. The term, $p$, takes the values of one or two for single-polarization or polarization-multiplexed 
signals, respectively. Finally, $\sigma_{n s-n p}^{2}$ may be approximated using the rectangular filter approach [24] from directly-detected systems as:

$$
\sigma_{n s-n p}^{2}=4 \mathrm{R}^{2} N_{s} N_{p} W_{p} R_{s}
$$

With Equations (2-5), one may estimate the SNR for SDM-SHD as [21]:

$$
\mathrm{SNR}_{S H}=\frac{2 B_{\mathrm{ref}}}{p R_{s}} \mathrm{OSNR}_{s}\left(1+\frac{W_{p}}{2 p R_{s}} \frac{\mathrm{OSNR}_{s}}{\mathrm{OSNR}_{p}}+\frac{W_{p}}{2 B_{\mathrm{ref}} \mathrm{OSNR}_{p}}\right)^{-1}
$$

where $P_{s}, \mathrm{OSNR}_{s}$ and $\mathrm{OSNR}_{p}$ are the average signal power, the signal OSNR and the PT OSNR, respectively. $B_{\text {ref }}$ is the reference bandwidth used for the OSNR measurement. Note also that the SNR for an equivalent ID detection system with identical signal OSNR, $\mathrm{OSNR}_{I D}=\mathrm{OSNR}_{s}$, is given by [23]:

$$
\mathrm{SNR}_{I D}=\mathrm{OSNR}_{I D} \frac{2 B_{\mathrm{ref}}}{p R_{s}}
$$

From Equations (6) and (7), one may easily compute the OSNR penalty of SHD systems with respect to ID for QPSK signals as the ratio between $\mathrm{OSNR}_{S}$ and $\mathrm{OSNR}_{I D}$ to reach $\mathrm{SNR}_{S H}=\mathrm{SNR}_{I D}$, in linear units, as:

$$
\Delta \mathrm{OSNR}=\left.\frac{\mathrm{OSNR}_{s}}{\mathrm{OSNR}_{I D}}\right|_{\mathrm{SNR}_{S H}=\mathrm{SNR}_{I D}}=1+\frac{W_{p}}{2 p R_{s}} \frac{\mathrm{OSNR}_{s}}{\mathrm{OSNR}_{p}}+\frac{W_{p}}{2 B_{\mathrm{ref}} \mathrm{OSNR}_{p}}
$$

Equation (8) provides a general model for the OSNR penalty of SHD systems with respect to ID systems with arbitrary signal and PT power and OSNR and for single and dual-polarization QPSK signals. It has also been shown to provide a reasonable approximation for the case of square QAM signals [22]. For moderate values of $\mathrm{OSNR}_{p}$, the third term on the right-hand side of Equation (8) may be neglected, in which case, the SNR penalty becomes mostly dependent on the term, $W_{p} / R_{s}$, referred to as the normalized PT bandwidth [21], and the ratio between the OSNR of the signal and PT.

To adapt the result in Equation (8) to the case of PDM-SHD systems, one needs to consider that measured OSNR is dependent on the signal and PT average powers as [21]:

$$
\mathrm{OSNR}_{S H}=\frac{P_{s}+P_{p}}{2 B_{\mathrm{ref}} N_{s}}=\mathrm{OSNR}_{s}+\mathrm{OSNR}_{p}
$$

where $\mathrm{OSNR}_{s}$ and $\mathrm{OSNR}_{p}$ now become the OSNR of the signal and PT components separately, with single-polarization ASE noise. Hence, as in [20], Equation (8) may be rewritten as:

$$
\Delta \mathrm{OSNR}=1+\frac{P_{p}}{P_{s}}+\frac{W_{p}}{2 R_{s}}\left(1+\frac{P_{s}}{P_{p}}\right)
$$

Note that Equation (9) replaces the dependence on the ratio between the signal and PT OSNRs by the ratio between the signal and PT average powers. Ref.25 should appear after ref. 24

Equations (8) and (10) have been experimentally verified in [21,22], for PDM-SHD and SDM-SHD systems, respectively. However, it can be shown that the validity of this model is preserved for more general systems. For example, in digital self-homodyne detection [25], described in Section 3.2.2, and shared carrier reception SDM systems [26], described in Section 4.1, digital processing is used to significantly reduce the PT filter bandwidth and, consequently, the penalty of SHD systems. 


\subsection{DSP Savings and Phase Noise Cancellation}

A strong advantage of using SHD in optical communication systems is the reduction of the required DSP complexity at the receiver, which potentially enables significant energy savings compared to an equivalent ID transmission system although we note that energy savings are system dependent and depend largely on what percentage of DSP resource energy is required to mitigate other impairments, such as chromatic dispersion [27,28]. The origin of DSP savings from using SHD is two-fold. Firstly, since the LO and signal are derived from the same laser and assuming that any path difference is orders of magnitude less than the laser coherence length, then no LO-transmitter laser frequency offset needs to be estimated and compensated for $[12,13,28]$; secondly, the reduction of the total system phase noise enables the carrier phase $(\mathrm{CP})$ to be estimated at a significantly reduced frequency than required in an ID receiver. The origin of these savings is evident in Figure 2, which shows the estimated and unwrapped carrier phase evolution for a single polarization QPSK signal at 25 Gbaud, transmitted through a $10.1 \mathrm{~km} \mathrm{MCF}$. The figure shows a $200-\mu$ s span containing $2 \times 10^{6}$ symbols using both an external cavity laser (ECL) and distributed-feedback DFB laser transmitters with the linewidth measured to be $730 \mathrm{KHz}$ and $3 \mathrm{MHz}$ at $193.6 \mathrm{THz}$, respectively [29]. Measurements were taken for SHD with path lengths closely aligned and then with a PT decorrelated from the signal by bypassing the MCF to approximate ID. To estimate the CP, the Viterbi and Viterbi (V\&V) algorithm [28] was used, carrying out the carrier-phase estimation on blocks of 128 samples (64 symbols) each.

Figure 2. Carrier phase evolution over two million symbols for path length aligned and misaligned SDM-SHD for both the external cavity laser (ECL) and DFB laser transmission.

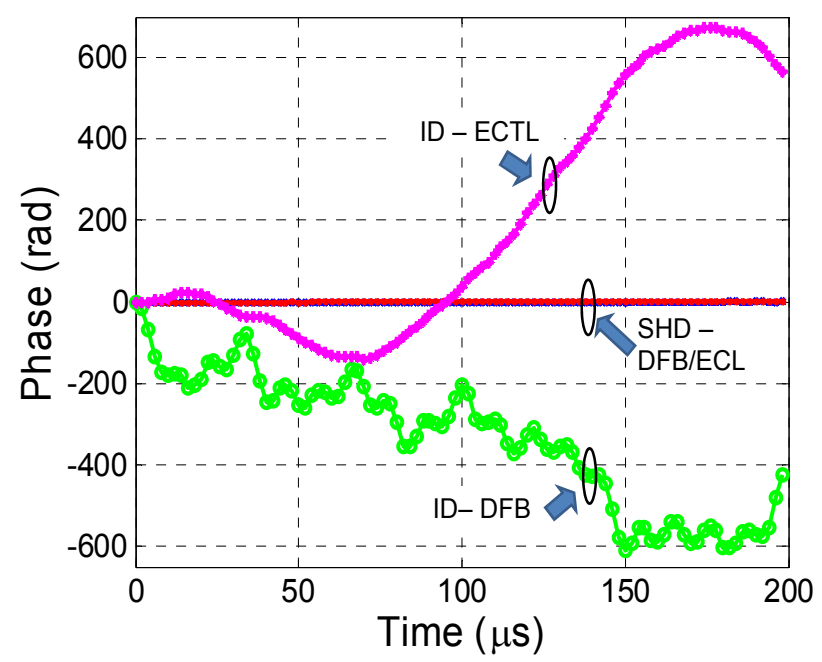

From Figure 2, it is evident that in the SHD case, even in the presence of the various noise sources, including inter-core crosstalk, only a residual CP correction is required for both laser types. This result qualitatively verifies that the laser phase noise is cancelled when employing SHD with good path alignment at the receiver [30]. With the path length misaligned by the $10.1 \mathrm{~km}$ span, the receiver behaves as in the ID case, and phase wanderings of over a $\pm 600 \mathrm{rad}$ range can be observed for both lasers. Furthermore, we note that the phase variations of the narrower linewidth ECL appear smoother than the DFB case, suggesting that their use with ID would reduce the likelihood of phase slips. 
The performance penalty of reducing the $\mathrm{CP}$ estimation rate was also investigated by BER measurements in [12,13] with 25 Gbaud, QPSK signals using SDM-SHD in a 19 core fiber. In this study, experimental data was received in a sampling oscilloscope and reprocessed with the V\&V algorithm [28] used in CP recovery, and the unwrapped CP estimation buffer was updated only once every $\mathrm{N}$ blocks. Consequently, the same estimated $\mathrm{CP}$ angle is used for $\mathrm{N}$ consecutive blocks, as shown in the diagram of Figure 3. All other variable estimators, such as normalization, CD compensation and CMA equalization, were computed on a block-by-block basis.

Figure 3. Block processing chart for the carrier phase estimation algorithm.
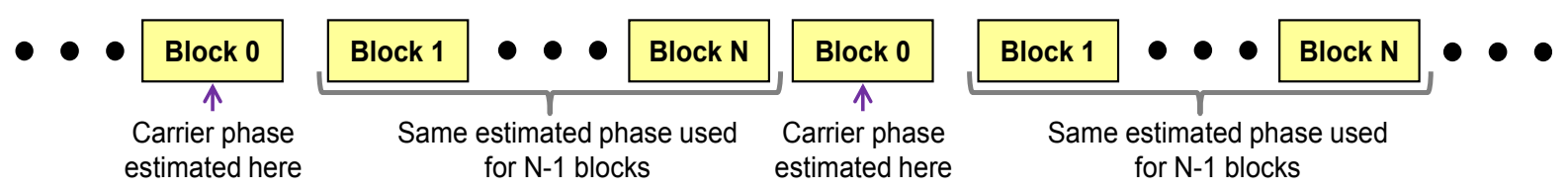

Figure 4 shows the average BER as a function of the number of skipped blocks $(\mathrm{N})$ for $\mathrm{V} \& \mathrm{~V}$ estimation block sizes of 32, 64 and 128 samples, for both the SHD (solid lines) and the ID (dashed lines). Figure 4 shows that for low values of N (high CP estimation update rates), longer estimation sequences perform better, since more noise is removed by the longer $V \& V$ estimators. As the estimation update rate decreases (larger $\mathrm{N}$ ), the performance degrades more rapidly for longer estimators. This is due to the fact that a longer estimator is less able to track the phase changes due to the residual linewidth, and thus, the probability of cycle slips increases [31]. However, up to a value of $\mathrm{N}=10^{3}$, the BER penalty is less than $2 \times 10^{-6}$ for all the three estimation block sizes considered with SHD. In the ID case, the impact of the laser linewidth is evident with cycle slips after less than 10 skipped blocks, with the more adaptive shorter estimators performing slightly better as $\mathrm{N}$ increases. Hence, Figure 4 shows that SHD is able to reduce the required frequency of carrier phase recovery by three orders of magnitude.

Figure 4. Averaged BER as a function of the carrier phase (CP) estimation rate for estimation block sizes of 32, 64 and 128 samples and aligned pilot-tone (PT) and signal paths (solid lines) and misaligned PT and signal paths (dashed lines).

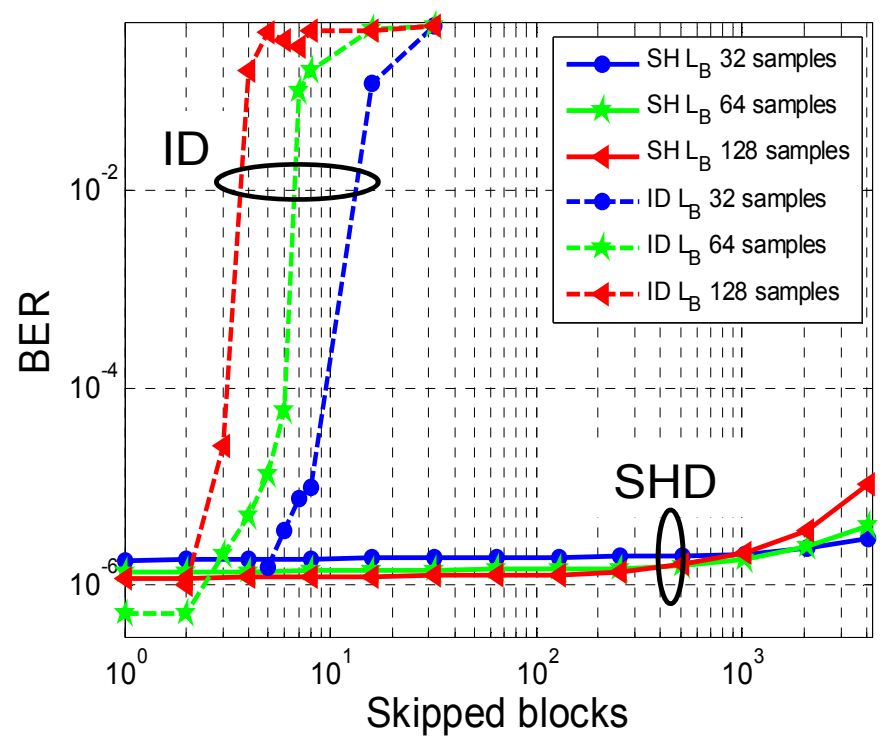




\subsection{Requirement for Receiver Polarization Control of Pilot-Tone}

An implementation problem in SHD systems is the polarization alignment of the LO and signal at the input to the coherent receiver optical signal mixer. In polarization diverse ID systems, the state of polarization (SOP) of the locally-generated LO can be easily aligned to the optical axis of the PBS of the optical hybrid, ensuring data recovery regardless of the incoming signal polarization state. However, for SHD systems, since the SOP of the PT after transmission is unknown and time-varying, this condition must be achieved by tracking and/or controlling the SOP of the PT before the optical hybrid. For PDM-SHD systems, assuming the polarization-orthogonality is maintained after transmission, the signal and PT may be separated by a PBS only if the input SOP is correctly aligned with the PBS optical axis and constant over time. Similarly for SDM-SHD systems, the SOP of the PT must be aligned with the SOP of the signal for single polarization data or at an angle of 45 degrees with respect to the optical axis of the optical hybrid for polarization diverse receivers.

Although not yet demonstrated specifically with SHD systems, active polarization tracking and control systems have been investigated for more than 25 years, and it is envisaged that they could be applied to SHD systems. The first scheme [32] used fiber squeezers and integrated optical components to control the SOP based on feedback from a PC. Subsequently, lithium-niobate polarization transformers [33,34] have been used to improve performance, and experimental demonstrations have reached tracking speeds of up to $50 \mathrm{krad} / \mathrm{s}$ for QPSK transmission [35] and with 112Gb/s PDM-RX-DPSK signals [36] and endless polarization tracking at $40 \mathrm{krad} / \mathrm{s}$ with 20Gb/s PDM-RZ-DPSK signals [37] and with additional phase control [34]. In addition, it may also be possible to use passive components to split the PT into polarization components using a PBS aligned to the desired SOP and apply a phase shift to one component before recombination. However, such a scheme requires an accurate and constant path length to ensure constructive interference between the two paths and will also not work in the presence of polarization mode-dispersion (PMD) arising from birefringence in the transmission fiber. It is for this reason that SHD systems used for long distance transmission will most likely require active polarization tracking and adjustment before the receiver if such techniques can be sufficiently fast to track SOP fluctuations.

\subsection{Other Pilot-Tone Transmission Schemes}

A variant of SHD, which is compatible with both polarization and space division multiplexing, is the transmission of pilot symbols between data symbols. In these schemes, un-modulated pilot symbols are time-domain multiplexed with data. SHD is achieved by mixing the incoming data signal with versions of itself delayed by one or more bit-slots, to match up data symbols with pilot-symbols at the receiver. Such schemes may be considered analogous to differential detection and have been demonstrated successfully with up to four data symbols for each PT symbol to provide SHD with a $20 \%$ overhead [38] and at data rates up to $40 \mathrm{~Gb} / \mathrm{s}[39,40]$ without the requirement for optical phase locked loops, high speed electronic circuits or complex coding schemes used in other homodyne detection schemes [41].

Although classified as heterodyne detection and, therefore, not the focus of this paper, significant advances in the transmission of high-order QAM formats has been achieved by transmitting data with 
a PT obtained by frequency shifting the unmodulated carrier [42-45]. The PT is used as a phase reference to a PLL locking a tunable tracking laser used as the LO at the receiver and allows the use of both polarizations for signal data at the expense of a small increase in the required signal bandwidth. This scheme has allowed the transmission of signals up to 512 QAM [44], as well as 256 QAM-OFDM [45] with high spectral efficiency, but does not allow phase noise cancellation and the linewidth tolerance characteristic of the schemes described in Sections 3 and 4.

\section{PDM-SHD: Implementations and Developments}

\subsection{PDM-SHD Transmission and Advanced Modulation Formats}

Following on from the early work of Miyazaki and Kubota [1,2] polarization-multiplexed PTs were used to enable transmission and investigation of advanced modulation formats and led to the development of a new variant of modulator to provide a signal and PT from a single polarization laser input, referred to as a pilot-tone vector modulator (PTVM) [46]. The first transmission results used $20 \mathrm{~Gb} / \mathrm{s}$ QPSK signals with high phase-noise transmitters, including a spectrum sliced ASE light source $[5,6]$ or a Fabry-Perot diode laser [7]. The PTVM enabled a series of measurements with high-order QAM and multi-level phase modulation formats. Sixteen-QAM modulation was demonstrated up to $40 \mathrm{~Gb} / \mathrm{s}$ with real-time modulation and de-modulation [47] and with inter-symbol-interference suppression with pre-equalized driving signals [48]. The same technique was used to transit 5 Gbaud 64 QAM over $60 \mathrm{~km}$ without dispersion compensation [8], and phase modulated formats included eight-PSK at $30 \mathrm{~Gb} / \mathrm{s}$ [49,50]. Sixteen-QAM modulation was also used to investigate the tolerance to non-linear impairments in SHD transmission systems over a 116-km link [3].

\subsection{Developments of PDM-SHD}

\subsubsection{Spectral Interleaving of Signal/PT}

A useful technique to reduce the SE cost of PDM-SHD schemes is the spectral interleaving of the signal and PT $[14,15]$. As shown in schematic form in Figure 5, in this scheme, both polarizations are used to transmit data signals with a frequency shift applied to one polarization to allow the transmission of the spectrally narrow pilot-tone between WDM channels on the orthogonal polarization.

Figure 5. PT and signal relationship for PDM-SHD and PT-interleaved PDM-SHD.

(a)

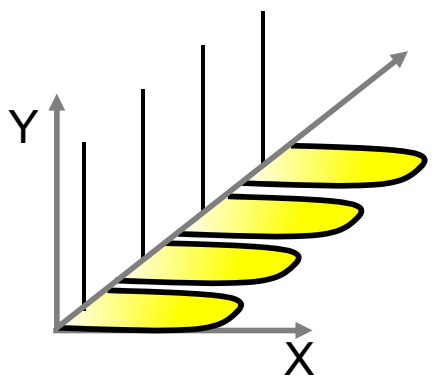

(b)

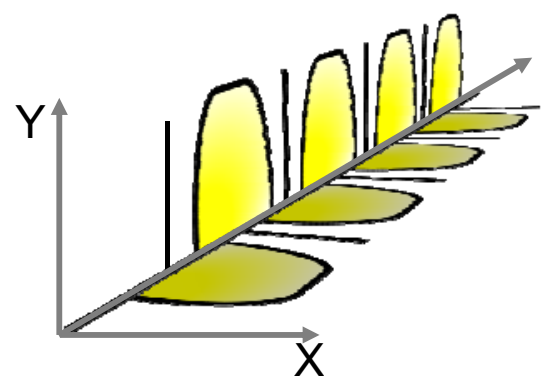

Compared to an SHD system transmitting data on a single polarization, this technique has been shown to provide $33 \%$ improvement for five wavelength channels carrying $10 \mathrm{~Gb} / \mathrm{s} \mathrm{NRZ} \mathrm{signals} \mathrm{[15],}$ 
and simulations of the same set-up suggested that use of a $0.1 \mathrm{GHz}$ PT filter could improve this to $77 \%$, approaching that of ID PDM transmission.

\subsubsection{Digital Self-Homodyne Detection}

As outlined in Section 2.4, coherent detection of polarization multiplexed PT signals requires accurate tracking of the polarization of the PT at the receiver input, which may limit their practicality and increases the complexity of the receiver. As an alternative, digital SHD has been proposed for PDM-SHD [25] and a similar scheme, discussed in Section 4.1, implemented in SDM systems [26]. This approach receives both signal and PT components with a polarization-diverse coherent ID receiver front-end, as shown in Figure 6. After the ID front-end, both components are converted to the digital domain, where they can be digitally aligned. After polarization alignment and assuming perfect dispersion compensation at the ID front-end, the signal and PT components can be approximated by:

$$
\begin{aligned}
& r_{s}=e_{s} \cdot e_{l o}^{*} \\
& r_{p}=e_{p} \cdot e_{l o}^{*}
\end{aligned}
$$

where $e_{s}, e_{p}$ and $e_{l o}$ are the complex envelopes of the signal, PT and LO from the ID front-end. Note that $r_{s}$ and $r_{p}$ are intermediate frequency (IF) signals, which are random and time-varying, arising from the frequency offset between the transmitter and LO lasers. From Equation (11), the original signal may be recovered with a single complex multiplication as:

$$
r=r_{s} \cdot r_{p}^{*}=e_{s} \cdot e_{p}^{*} \cdot\left|e_{l o}\right|^{2}
$$

Equation (12) shows that the recovered signal no longer includes the LO phase noise, thus preserving the phase noise cancellation characteristic of SHD systems. Consequently, this scheme allows the use of high linewidth lasers for the generation of the signal and PT, as well as the LO.

Figure 6. Layout for digital self-homodyne detection.

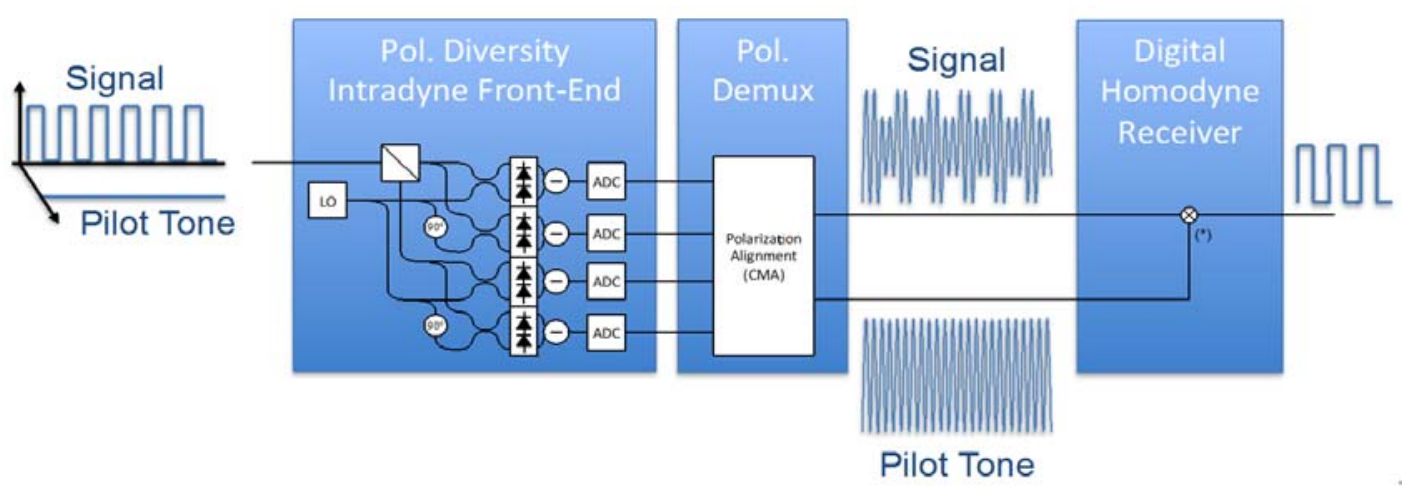

\section{SDM- SHD: Implementations and Developments}

The recent advancement of SDM technology as a means of meeting the expected growth in the capacity requirement for the next generation of optical networks has also enabled the possibility of sharing the SE cost of SHD between many spatial channels. As described in Section 3.2.1, even with spectral interleaving techniques, using polarization multiplexed PTs still has a strong impact on SE. 
By transmitting the PT in one SDM channel and using the remaining channels for signal transmission, the spectral efficiency cost is inversely proportional to the number of SDM channels. For example, using a 19-core fiber results in a 5.3\% reduction of spectral efficiency compared to an equivalent system with ID, although this figure rises to $14.3 \%$ for a seven-core fiber. Although SHD may also be compatible with multi-mode transmission, the impact of modal dispersion has not been investigated experimentally, and PT transmission in multi-mode fiber has only been investigated by simulation [51]. For this reason, all SDM-SHD experiments to date have been performed with MCF with each core diameter being similar to that of a single mode fiber. In an SDM system based on MCF, it is envisaged that different channels experience the same environmental disturbances. This was investigated for spatial super-channels where long-term differential phase drift was shown to vary more slowly with absolute variation $90 \%$ lower over 400 us [19]. Such a feature is advantageous for SHD, which relies on matching the path length between pilot-tone and information channels for strong phase noise cancellation. In this section, we outline transmission demonstrations using SHD and shared carrier reception schemes, techniques for in-service path length alignment and approaches to integrate $\mathrm{SH}$ transmission with networking and spatial super-channels.

\subsection{Transmission}

Early attempts to apply SHD to SDM systems were performed in a prototype 19-core fiber using a free space coupling system $[17,18]$ with the PT transmitted through one fiber core with data signals transmitted in the remaining cores. In this work [29,52], the feasibility of SHD was determined by noise-loading measurements to determine the penalty of SDM as a function of the WDM channel spacing for a different laser linewidth. An additional signal impairment in SDM systems is crosstalk from other SDM channels, and the impact of this was also studied by measuring the OSNR penalty of transmitting the PT in different fiber cores. As shown in Figure 7, compared to SHD without the presence of dummy signal channels in all cores, using the center core for PT transmission with signals in all cores results in a $1-\mathrm{dB}$ penalty due to the higher crosstalk from the increased number of neighboring cores. The penalty for using the outer core for PT transmission was under $0.5 \mathrm{~dB}$, showing that using a lower crosstalk outer-core for PT transmission is advantageous in an SDM systems.

SDM-SHD systems have been used to demonstrate high capacity transmission using broad linewidth DFB lasers. In [4], a 19-core fiber was used to demonstrate a $105 \mathrm{~Tb} / \mathrm{s}$ transmission system using low-cost DFB laser transmitters over $10.1 \mathrm{~km}$. This experiment showed the potential for high capacity transmission using SHD by using 125 individual DFB transmitters with frequencies between 189.1 and $195.3 \mathrm{THz}$ on a $50-\mathrm{GHz}$ grid with the linewidth varying from one to $5.3 \mathrm{MHz}$ and each carrying 25 Gbaud QPSK signals. Assuming a 7\% FEC overhead, the $105 \mathrm{~Tb} / \mathrm{s}$ transmission is equivalent to a SE of $16.7 \mathrm{~b} / \mathrm{s} / \mathrm{Hz}$. The BER was measured for all signal channels and cores and found to vary between $2 \times 10^{-4}$ and $2.7 \times 10^{-6}$, demonstrating error rates well below the $2 \times 10^{-3}$ limit of commercially available FEC systems [53]. The transmission capacity was then doubled to $210 \mathrm{~Tb} / \mathrm{s}$ $(\mathrm{SE}=33.4 \mathrm{~b} / \mathrm{s} / \mathrm{Hz})$ [54] by adopting polarization-division multiplexing QPSK signals, and the measured BERs for all cores, for five wavelengths, including the edge and center channels, is shown in Figure 8. 
Figure 7. Measurement of SDM penalties when transmitting the pilot-tone on different fiber cores compared to SHD transmission without dummy channels. OSNR, optical signal-to-noise ratio.

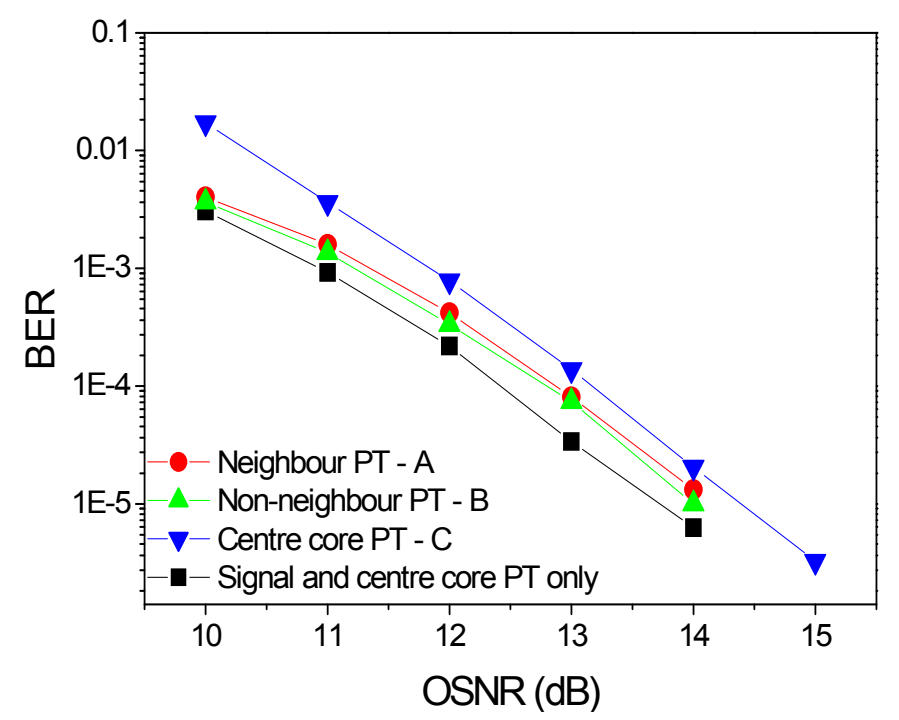

Figure 8. Measured BER in each signal core for five channels with equally spaced optical frequencies ranging from $195.3 \mathrm{THz}$ (Ch. 1) to $189.1 \mathrm{THz}$ (Ch. 125).

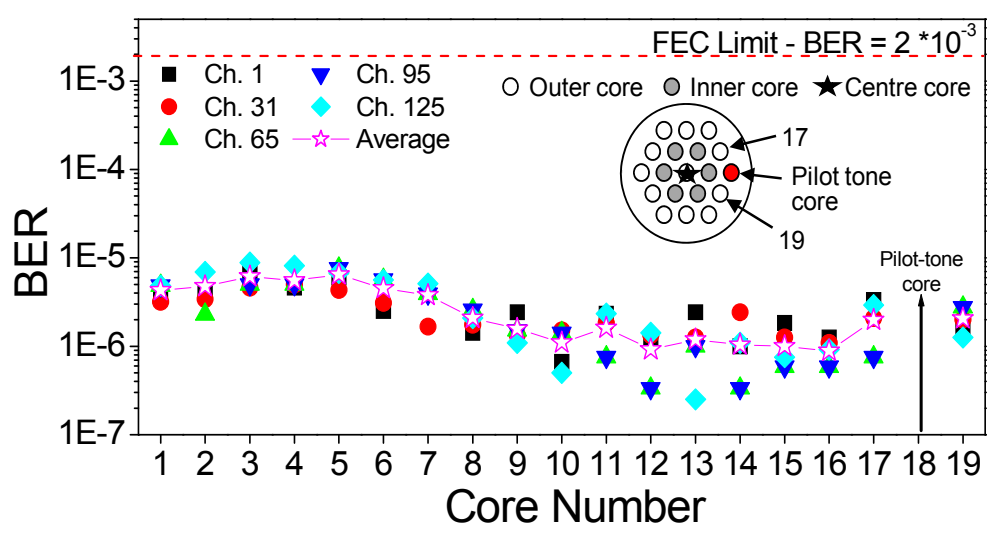

A technique similar to the digital self-homodyne detection described in Section 3.2.2 has also been applied to MCF systems. This shared carrier reception (SCR) scheme [26] also relies on the transmission of an unmodulated pilot-tone, tapped from the same laser as signals in a dedicated fiber core, but instead of using this as the LO for coherent reception, the SCR-PT is received by an ID coherent receiver. The precise frequency and carrier phase information extracted from DSP [55] is then used to simplify the DSP of the received signal cores. As with digital SHD, if the LO laser is sufficiently well tuned, then digital domain filtering may be used to improve the transmission characteristics. In [24], a 0.5-dB improvement in the Q-factor was observed and a tolerance of up to $6 \mathrm{GHz}$ to $\mathrm{LO} /$ signal detuning with the path length tolerance dependent on the bandwidth of the electrical filter. This technique was used for a long distance transmission of $10 \times 128 \mathrm{~Gb} / \mathrm{s} 16$ QAM signals with $25 \mathrm{GHz}$ spacing [56] over a distance of $1.815 \mathrm{~km}$ using $100 \mathrm{kHz}$ linewidth lasers with phase noise cancellation, allowing a $1,650 \mathrm{~km}$ transmission for a 1-MHz linewidth DFB laser, an increase of $165 \mathrm{~km}$ from a SCR receiver, with frequency offset correction only. 


\subsection{Moves towards Networking}

In addition to a point-to-point transmission demonstration, the first experiments exploring the feasibility of SHD from a networking perspective have also been demonstrated. Previously, architecture-on-demand (AoD) nodes have been widely proposed and also demonstrated to incorporate SDM links [57,58], and reconfigurable add-and-drop-multiplexers (ROADM) designs for the switching of spatial super-channels [19,59] in MCFs have also been proposed. In [60], a reconfigurable SDM-WDM hybrid add-drop node capable of routing both signals and PTs was demonstrated for $100 \mathrm{~Gb} / \mathrm{s}$ PDM-QPSK signals. Figure 9 shows a schematic of the SDM-WDM add and drop node used in this demonstration.

Figure 9. Add and drop in the SDM node with PT. MCF, multi-core fiber.

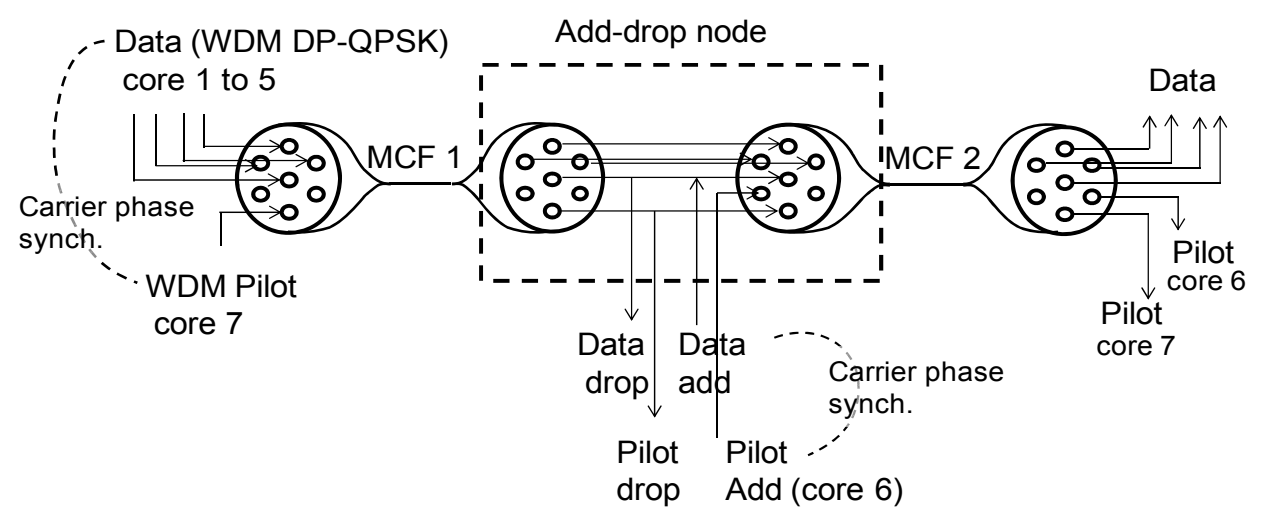

SHD has also been used in a larger network demonstration utilizing AoD nodes and softwaredefined networking (SDN). In this demonstration, both seven-core and 19-core MCFs were used in a network demonstration using spatial super-channels and a variety of modulation formats with both signal and PTs dynamically assigned by an SDN controller over an open flow interface [61,62]. This demonstration showed that where capacity allows, the advantages of SHD may be exploited even in dynamic networking scenarios by integrating signal and PT management into control plane architectures.

\subsection{Path Length Alignment Scheme}

Phase noise cancellation has been shown in the previous sections to be an essential property of SHD systems. However, optimum performance requires some degree of alignment of the signal and PT path lengths from the point of separation at the transmitter side, up to the balanced photodetectors at the receiver side. The required path length accuracy is currently unclear, being modulation format- and system-dependent. It was estimated to be $10 \%$ of the bit-period for PDM-SHD in the presence of non-linearity [3]; yet, measurements with moderate launch power in MCFs [12,13,52] showed that removal of tens of meters of fiber had a minimal impact on BER. Hence, the required path length accuracy for different conditions is the subject of current work, although techniques to minimize it have been investigated, as described below.

The residual phase noise resulting from path-length misalignment in SHD systems may be modeled by extending the complex envelopes of the signal and PT in Equation (1), to give: 


$$
i_{r}(t)=2 R \sqrt{P_{s} P_{p}} s(t) e^{j \Delta \phi(t)}
$$

where $s(t), P_{s}$ and $P_{p}$ are the normalized information signal and the average signal and PT powers, respectively. The residual phase noise in SHD is modeled by the term $\Delta \phi(t)=\phi(t)-\phi(t-\tau)$, with $\phi(t)$ as the phase noise of the transmission laser. The term, $\tau$, is the delay between the signal and PT due to path-length misalignment. To model the residual phase noise, one may assume that $\mathrm{f}(\mathrm{t})$ can be modeled as a Wiener process with variance increasing linearly with the observation interval. Hence, the variance of $\Delta \phi(t)$ can be obtained by restricting the observation interval to the propagation delay between signal and PT. As such, for small path length delays, $\tau<t$ the residual phase noise has a constant variance [63] given by:

$$
\sigma_{\Delta \phi}^{2}=2 \pi \Delta l|\tau|
$$

where $\Delta l$ is the linewidth of the transmission laser. Equation (14) shows that the variance of the residual phase noise is directly proportional to the propagation delay between signal and PT. Furthermore, it shows that for very large misalignment, the variance of the phase noise in a SHD system becomes similar to that obtained in an ID system with uncorrelated signal and PT, similar to the experiment described in Section 2.3. Equation (14) provides a basis for the design of DSP for phase noise estimation and compensation in SHD systems, considering a misalignment margin between the signal and PT path lengths.

In addition to laser phase noise, appropriate path-length alignment can also cancel or reduce the impact of nonlinear phase noise introduced by fiber nonlinearities in polarization-multiplexed PT systems [3]. In this case, it has been shown that, in a first order approach, the nonlinear residual nonlinear phase noise in SHD systems over one fiber span is approximated by [3]:

$$
\Delta \phi_{\mathrm{NL}}(t) \approx \gamma\left(P_{s}+P_{p}\right) L_{\mathrm{eff}}\left[|s(t)|^{2}-|s(t-\tau)|^{2}\right]
$$

where $\gamma$ and $L_{\text {eff }}$ are the fiber nonlinearity coefficient and its effective length, respectively. Note that, unlike laser phase noise, the nonlinear phase noise varies at a rate similar to the symbol rate, and its compensation via DSP is generally harder to achieve. Hence, accurate path-length monitoring and alignment in these systems is critical in achieving acceptable performance [3].

In realistic implementations of SDM or PDM-SHD, path length alignment has been achieved using variable delay lines in the signal or PT paths, at the transmitter or receiver sides $[4,22,52,54,61,62]$. The variable delay lines may be controlled using path-length alignment monitoring subsystems, such as the one proposed in [30]. Figure 10 shows the generic scheme for this subsystem. It is based on modulating the phase of the light wave with a low frequency tone prior to separation of the signal and PT paths. Upon recombination at the at the receiver side, non-zero path-length misalignment will yield a measureable interference signal, as shown in the inset of Figure 10. This signal can be used as feedback to control a variable optical delay line. When perfect path-length alignment is achieved, the interference signal is canceled and has no impact on the system performance.

Figure 11 shows the dependence of the interference signal power on the coordinate of the variable delay line for two cores of a 19-core SDM system using SHD and considering modulation frequencies of $70 \mathrm{MHz}$ and $160 \mathrm{MHz}$ [30]. The path-length monitoring subsystem provides resolutions of 
$2 \mathrm{~dB} / \mathrm{cm}$ and $1 \mathrm{~dB} / \mathrm{cm}$ for $70 \mathrm{MHz}$ and $160 \mathrm{MHz}$ modulation frequencies, respectively, which effectively allow sub-centimeter path-length alignment. Alternatively, an SCR scheme, where signal and PT are independently detected, allows path-length monitoring and alignment fully performed in the digital domain $[26,56]$. This facilitates the practical implementation of such systems, by avoiding the need for optical delay lines and feedback mechanisms, despite the underlying cost of the shared PT receiver.

Figure 10. Path-length alignment monitoring subsystem. LO, local oscillator.

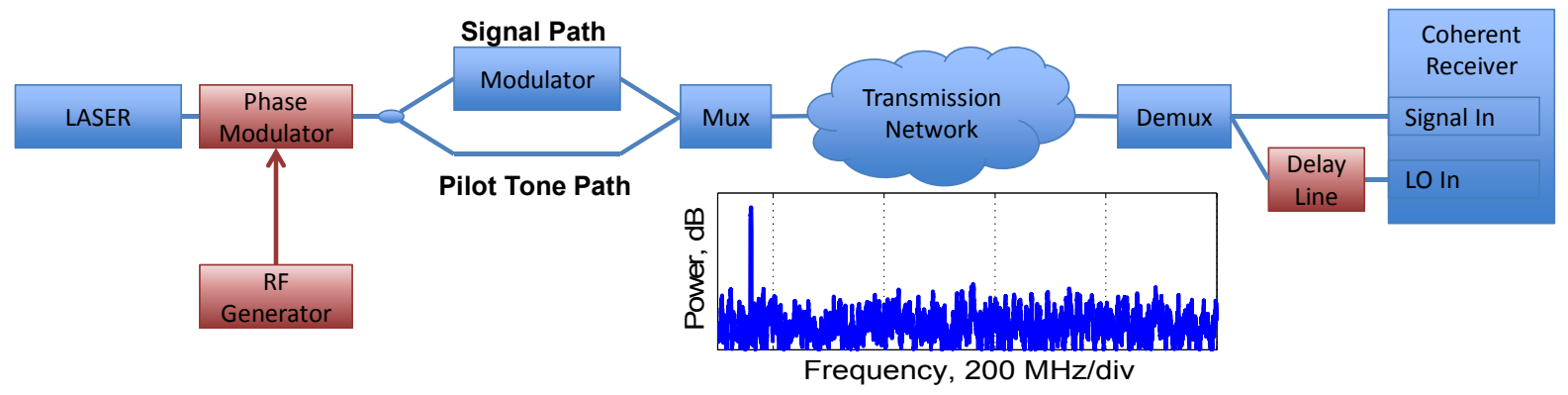

Figure 11. The dependence of the interference signal power on the coordinate of the variable delay line for path-length alignment monitoring.

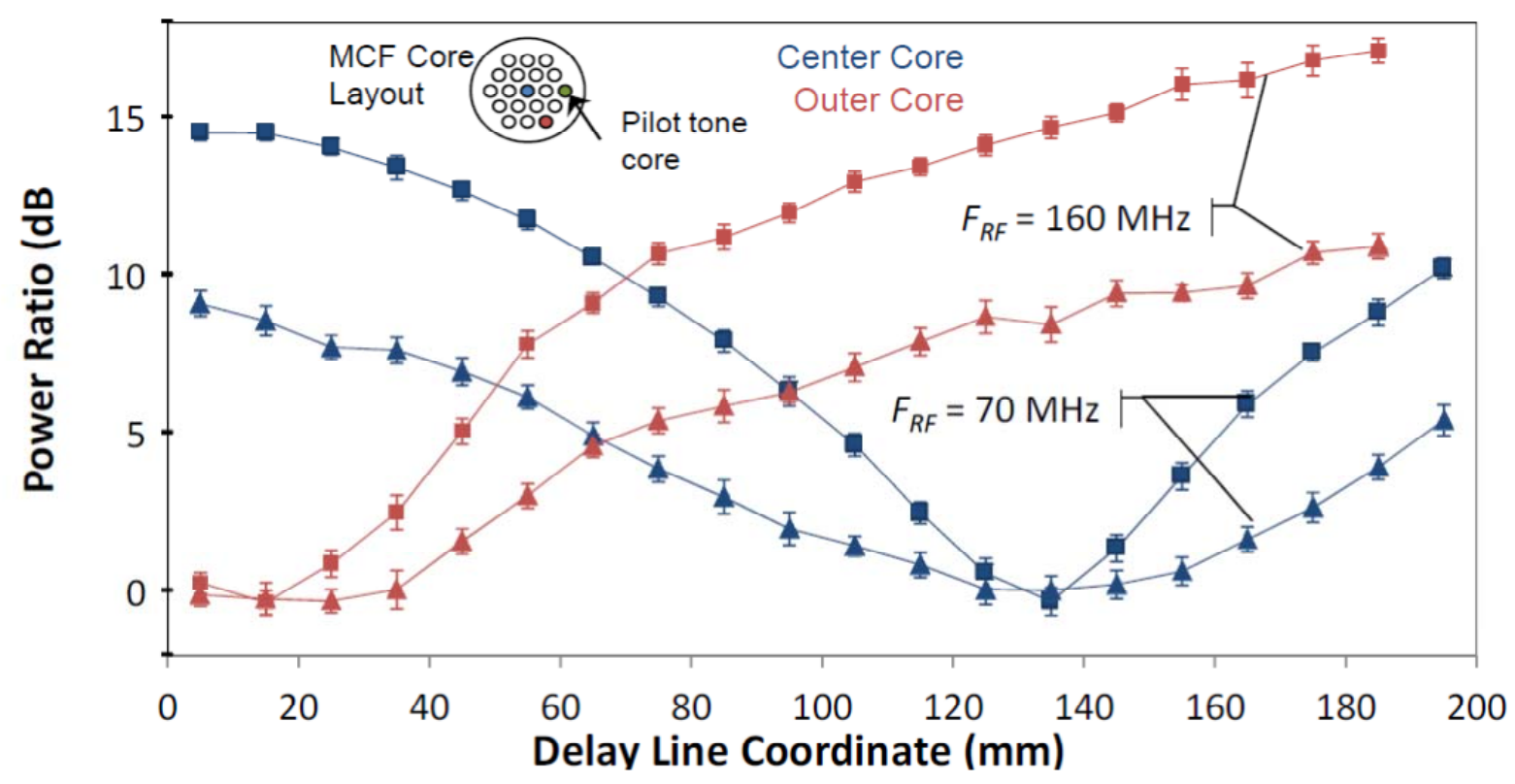

\section{Summary and Future Challenges}

The work summarized in this paper shows that self-homodyne detection has been well studied in optical transmission links for point-to-point communication in optical fiber systems with clear advantages and disadvantages. Compared to equivalent ID systems, pure SHD, as outlined in Sections 2.1 and 2.2, results in both a penalty in the required OSNR and a reduction in the achievable spectral efficiency. The spectral efficiency cost is more severe for a polarization multiplexed pilot-tone (PT), where it is typically 50\%, reducing to $33 \%$ using spectral interleaving techniques. When the PT is space-division multiplexed (SDM), the spectral efficiency reduction is inversely proportional to the number of SDM channels when either SHD or shared carrier reception techniques are used. The OSNR 
penalty may be well described and depends crucially on the bandwidth of the pilot-tone optical filter, typically varying between $2 \mathrm{~dB}$ and $4 \mathrm{~dB}$ in experimental investigations.

The advantages of SHD have also been well demonstrated. Phase noise cancellation leads to low susceptibility to phase noise originating from low-cost lasers, which is particularly advantageous when using phase-noise sensitive formats, such as high-order QAM modulation, commonly proposed to improve spectral efficiency, or OFDM. Additionally, we have seen that the energy and complexity cost of receiver digital signal processing can be minimized by using both SDM-SHD and shared carrier reception schemes.

The remaining challenges of SHD research lie in identifying the limits of transmission and in the implementation of the required subsystems, such as polarization alignment schemes. Although SCR schemes have been demonstrated with recirculating transmission, pure SHD has not yet been experimentally demonstrated beyond a point-to-point link. Verifying that phase noise cancellation and its benefits can be maintained in noise limited long-haul transmission links is crucial to proving the practical usefulness of SHD in optical transmission systems. Similarly, the precise level of path length alignment required to obtain the benefits needs to be studied in noise limited links, particularly in the presence of fiber non-linearity and for high-order modulation formats for which SHD is proposed to solve the phase noise dependence. Whether phase noise cancellation can be maintained and whether the required path length accuracy is achievable over the desired transmission lengths will ultimately determine whether self-homodyne detection will be a useful technique for future optical communication systems.

\section{Acknowledgments}

We acknowledge the support of NICT technical staff for many of the experiments described.

\section{Authors Contributions}

Benjamin J. Puttnam; Ruben S. Luís and José Manuel Delgado Mendinueta prepared the manuscript with feedback from all authors who also contributed to the work described as reflected in the references

\section{Conflicts of Interest}

The authors declare no conflict of interest.

\section{References}

1. Miyazaki, T.; Kubota, F. PSK self-homodyne detection using a pilot carrier for multibit/symbol transmission with inverse-RZ signal. IEEE Photonics Technol. Lett. 2005, 17, 1334-1336.

2. Miyazaki, T. Linewidth-Tolerant QPSK Homodyne Transmission Using a Polarization-Multiplexed Pilot Carrier. IEEE Photonics Technol. Lett. 2006, 18, 388-390.

3. Johannisson, P.; Sjödin, M.; Karlsson, M.; Tipsuwannakul, E.; Andrekson, P. Cancellation of nonlinear phase distortion in self-homodyne coherent systems. IEEE Photonics Technol. Lett. 2010, 22, 802-824. 
4. Puttnam, B.J.; Delgado-Mendinueta, J.M.; Sakaguchi, J.; Luis, R.S.; Klaus, W.; Awaji, Y.; Wada, N.; Kanno, A.; Kawanishi, T. 105 Tb/s Transmission System Using Low-cost, MHz Linewidth DFB Lasers Enabled by Self-Homodyne Coherent Detection and a 19-Core Fiber. In Proceedings of the Optical Fiber Communication Conference and Exposition and the National Fiber Optic Engineers Conference (OFC/NFOEC), Anaheim, CA, USA, 17-21 March 2013.

5. Nakamura, M.; Kamio, Y.; Lu, G.-W.; Miyazaki, T. Ultimately phase-noise tolerant QPSK homodyne using a spectrum-sliced ASE light source. IEICE Electron. Express 2007, 4, 406-410.

6. Nakamura, M.; Kamio, Y.; Lu, G.-W.; Miyazaki, T. Ultimate Linewidth-Tolerant 20-Gbps QPSK-Homodyne Transmission Using a Spectrum-Sliced ASE Light Source. In Proceedings of the Optical Fiber Communication and the National Fiber Optic Engineers Conference, OFC/NFOEC 2007, Anaheim, CA, USA, 25-29 March 2007.

7. Nakamura, M.; Kamio, Y.; Miyazaki, T. QPSK-Homodyne Transmission using a Multi-Wavelength Fabry-Perot Laser Diode. In Proceedings of the Lasers and Electro-Optics Conference, Baltimore, MD, USA, 6-11 May 2007.

8. Nakamura, M.; Kamio, Y. Linewidth-tolerant, ISI-suppressed 15-Gbit/s 64-QAM transmission over 120-km SSMF. In Proceedings of the 34th European Conference on Optical Communication, ECOC 2008, Brussels, Belgium, 21-25 September 2008.

9. Nakamura, M.; Kamio, Y. 30-Gbps (5-Gsymbol/s) 64-QAM Self-Homodyne Transmission over 60-km SSMF Using Phase-Noise Cancelling Technique and ISI-Suppression Based on Electronic Digital Processing. In Proceedings of the Optical Fiber Communication 2009, San Diego, CA, USA, 22-26 March 2009.

10. Kamio, Y.; Nakamura, M.; Miyazaki, T. 80-Gb/s 256-QAM signals using phase noise and DGD-tolerant pilot-carrier-aided homodyne detection. In Proceedings of the 33rd European Conference and Ehxibition of Optical Communication (ECOC), Berlin, Germany, 16-20 September 2007.

11. Luis, R.S.; Puttnam, B.J.; Mendinueta, J.-M.D.; Sakaguchi, J.; Shinada, S.; Kamio, Y.; Wada, N.; Nakamura, M. Self-homodyne coherent OFDM packet transmission without carrier frequency or common phase error estimation. In Proceedings of the IEEE 4th International Conference on Photonics, Malaka, Malaysia, 28-30 October 2013; pp. 123-125.

12. Delgado Mendinueta, J.M.; Puttnam, B.J.; Sakaguchi, J.; Luis, R.S.; Klaus, W.; Awaji, Y.; Kanno, A.; Kawanishi, T. Investigation of Receiver DSP Carrier Phase Estimation Rate for Self-Homodyne Space-Division Multiplexing Communication Systems. In Proceedings of the Optical Fiber Communication Conference and Exposition and the National Fiber Optic Engineers Conference (OFC/NFOEC), Anaheim, CA, USA, 17-21 March 2013.

13. Delgado Mendinueta, J.M.; Puttnam, B.J.; Sakaguchi, J.; Luis, R.S.; Klaus, W.; Awaji, Y.; Kanno, A.; Kawanishi, T. Energy Efficient Carrier Phase Recovery for Self-Homodyne Polarization-Multiplexed QPSK. In Proceedings of the 18th OptoElectronics and Communications Conference, Kyoto, Japan, 30 June-4 July 2013.

14. Sjödin, M.; Agrell, E.; Lu, G.-W.; Johannisson, P.; Karlsson, M.; Andrekson, P. Interleaved polarization division multiplexing in self-homodyne coherent WDM systems. In Proceedings of the 36th European Conference and Exhibition on Optical Communication (ECOC), Torino, Italy, 19-23 September 2010. 
15. Sjödin, M.; Agrell, E.; Johannisson, P.; Lu, G.-W.; Andrekson, P.A.; Karlsson, M. Filter optimization for self-homodyne coherent WDM systems using interleaved polarization division. IEEE J. Light. Technol. 2011, 29, 1219-1226.

16. Zhu, B.; Taunay, T.F.; Fishteyn, M.; Liu, X.; Chandrasekhar, S.; Yan, M.F.; Fini, J.M.; Monberg, E.M.; Dimarcello, F.V. 112-Tb/SDM DWDM transmission with 14-b/s/Hz aggregate spectral efficiency over a 76.8-km seven-core fiber. Opt. Express 2011, 19, 16665-16671.

17. Klaus, W.; Sakaguchi, J.; Puttnam, B.J.; Awaji, Y.; Wada, N.; Kobayashi, T.; Watanabe, M. Free-space coupling optics for multi-core fibers. In Proceedings of the IEEE Photonics Society Summer Topical Meeting Series, Seattle, WA, USA, 9-11 July 2012.

18. Sakaguchi, J.; Puttnam, B.J.; Klaus, W.; Awaji, Y.; Wada, N.; Kanno, A.; Kawanishi, T.; Imamura, K.; Inaba, H.; Mukasa, K.; et al. 19-core fiber transmission of $19 \times 100 \times 172-\mathrm{Gb} / \mathrm{s}$ SDM-WDM-PDM-QPSK signals at $305 \mathrm{~Tb} / \mathrm{s}$. In Proceedings of the National Fiber Optic Engineers Conference on Optical Fiber Communication Conference and Exposition (OFC/NFOEC), Los Angeles, CA, USA, 4-8 March 2012.

19. Feuer, M.D.; Nelson, L.E.; Zhou, X.; Woodward, S.L.; Isaac, R.; Zhu, B.; Taunay, T.F.; Fishteyn, M.; Fini, J.M.; Yan, M.F. Joint Digital Signal Processing Receivers for Spatial Superchannels. IEEE Photonics Technol. Lett. 2012, 24, 1957-1960.

20. Camatel, S.; Ferrero, V. Homodyne coherent detection of ASK and PSK signals performed by a subcarrier optical phase-locked loop. IEEE Photonics Technol. Lett. 2006, 18, 142-144.

21. Sjödin, M.; Johannisson, P.; Karlsson, M.; Tong, Z.; Andrekson, P.A. OSNR requirements for self-homodyne coherent systems. IEEE Photonics Technol. Lett. 2010, 22, 91-93.

22. Luis, R.; Puttnam, B.; Mendinueta, J.; Klaus, W.; Sakaguchi, J.; Awaji, Y.; Kawanishi, T.; Kanno, A.; Wada, N. OSNR Penalty of Self-Homodyne Coherent Detection in Spatial-Division-Multiplexing Systems. IEEE Photonics Technol. Lett. 2014, 26, 477-479.

23. Essiambre, R.; Kramer, G.; Winzer, P.J.; Foschini, G.J.; Goebel, B. Capacity Limits of Optical Fiber Networks. IEEE J. Light. Technol. 2010, 28, 662-701.

24. Rebola, J.; Cartaxo, A. Q-factor estimation and impact of 248 spontaneous-spontaneous beat noise on the performance of optically 249 preamplified systems with arbitrary optical filtering. IEEE J. Light. Technol. 2003, 21, 87-95.

25. Luis, R.S.; Puttnam, B.J.; Mendinueta, J.-M.D.; Sakaguchi, J.; Shinada, S.; Nakamura, M.; Kamio, Y.; Wada, N. Self-homodyne detection of polarization-multiplexed pilot tone signals using a polarization diversity coherent receiver. In Proceedings of the 39th European Conference and Exhibition on Optical Communication (ECOC 2013), London, UK, 22-26 September 2013.

26. Le Taillandier de Gabory, E.; Arikawa, M.; Hashimoto, Y.; Ito, T.; Fukuchi, K. A shared carrier reception and processing scheme for compensating frequency offset and phase noise of spacedivision multiplexed signals over multicore fibers. In Proceedings of the Optical Fiber Communication Conference and Exposition and the National Fiber Optic Engineers Conference (OFC/NFOEC), Anaheim, CA, USA, 17-21 March 2013.

27. Kuschnerov, M.; Bex, T.; Kainzmaier, P. Energy Efficient Digital Signal Processing. In Proceedings of the Optical Fiber Communication Conference and Exposition and the National Fiber Optic Engineers Conference (OFC/NFOEC), San Francisco, CA, USA, 9-13 March 2013. 
28. Savory, S.J. Digital Coherent Optical Receivers: Algorithms and Subsystems. IEEE J. Sel. Top. Quantum Electron. 2010, 16, 1164-1179.

29. Puttnam, B.J.; Sakaguchi, J.; Delgado-Mendinueta, J.M.; Klaus, W.; Awaji, Y; Wada, N.; Kanno, A.; Kawanishi, T. Investigating self-homodyne coherent detection in a 19 channel space-division-multiplexed transmission link. Opt. Express 2013, 21, 1561-1566.

30. Luis, R.S.; Puttnam, B.J.; Mendinueta, J.M.D.; Sakaguchi, J.; Klaus, W.; Awaji, Y.; Wada, N.; Kanno, A.; Kawanishi, T. In-service method of path length alignment in SDM systems with self-homodyne detection. In Proceedings of the 18th OptoElectronics and Communications Conference, Kyoto, Japan, 30 June-4 July 2013.

31. Meyr, H.; Moeneclaey, M.; Fechtel, S.A. Digital Communication Receivers: Synchronization, Channel Estimation, and Signal Processing; John Wiley \& Sons, Inc.: New York, NY, USA, 1997.

32. Noé, R.; Heidrich, H.; Hoffmann, D. Endless polarization control systems for coherent optics. IEEE J. Light. Technol. 1988, 6, 1199-1207.

33. Hidayat, A.; Koch, B.; Mirvoda, V.; Zhang, H.; Bhandare, S.; Ibrahim, S.K.; Sandel, D.; Noe, R. Fast Optical Endless Polarization Tracking with LiNbO3 Component. In Proceedings of the Optical Fiber communication/National Fiber Optic Engineers Conference, San Diego, CA, USA, 24-28 February 2008.

34. Koch, B.; Noé, R.; Mirvoda, V.; Sandel, D. 20 krad/s Endless Optical Polarisation and Phase Control. Electron. Lett. 2013, 49, 483-485.

35. Pfau, T.; Peveling, R.; Hoffinann, S.; Bhandare, S.; Ibrahim, S.; Sandel, D.; Adamczyk, O.; Porrmann, M.; Noe, R.; Achiam, Y.; et al. PDL-Tolerant Real-time Polarization-Multiplexed QPSK Transmission with Digital Coherent Polarization Diversity Receiver. In Proceedings of the Digest of the IEEE/LEOS Summer Topical Meetings, Portland, OR, USA, 23-25 July 2007.

36. Koch, B.; Noé, R.; Mirvoda, V.; Griesser, H.; Bayer, S.; Wernz, H. Record 59-krad/s Polarization Tracking in 112-Gb/s640-km PDM-RZ-DQPSK Transmission. IEEE Photonics Technol. Lett. 2010, 22, 1407-1409.

37. Koch, B.; Noé, R.; Mirvoda, V.; Sandel, D.; Jan, O.; Puntsri, K. 20-Gb/s PDM-RZ-DPSK Transmission with $40 \mathrm{krad} / \mathrm{s}$ Endless Optical Polarization Tracking. IEEE Photonics Technol. Lett. 2013, 25, 798-801.

38. Kamio, Y.; Miyazaki, T. Pilot-Symbol-Aided Self-Homodyne Detection for High-Efficiency Optical-Fiber Transmission System. In Proceedings of the Pacific Rim Conference on Lasers and Electro-Optics, CLEO/Pacific Rim 2005, Tokyo, Japan, 30 July-2 August 2005.

39. Lu, G.-W.; Nakamura, M.; Kamio, Y.; Miyazaki, T. 40-Gb/s QPSK with Inserted Pilot Symbols Using Self-Homodyne Detection. In Proceedings of the Optical Fiber Communication and the National Fiber Optic Engineers Conference, OFC/NFOEC 2007, Anaheim, CA, USA, 25-29 March 2007.

40. Lu, G.-W.; Nakamura, M.; Kamio, Y.; Miyazaki, T. 40-Gb/s QPSK and 20-Gb/s PSK with inserted pilot symbols using self-homodyne detection. Opt. Express 2007, 15, 7660-7666.

41. Kikuchi, K. Phase-diversity homodyne detection of multilevel optical modulation with digital carrier phase estimation. IEEE J. Sel. Top. Quantum Electron. 2006, 12, 563-570. 
42. Nakazawa, M.; Yoshida, M.; Kasai, K.; Hongou, J. $20 \mathrm{msymbol} / \mathrm{s}, 64$ and 128 QAM coherent optical transmission over $525 \mathrm{~km}$ using heterodyne detection with frequency-stabilised laser. Electron. Lett. 2006, 42, 710-712.

43. Nakazawa, M.; Hirooka, T.; Yoshida, M.; Kasai, K. Ultrafast Coherent Optical Transmission. IEEE J. Sel. Top. Quantum Electron. 2012, 18, 363-376.

44. Okamoto, S.; Toyoda, K.; Omiya, T.; Kasai, K.; Yoshida, M.; Nakazawa, M. 512 QAM (54 Gbit/s) coherent optical transmission over $150 \mathrm{~km}$ with an optical bandwidth of $4.1 \mathrm{GHz}$. In Proceedings of the 36th European Conference and Exhibition on Optical Communication, Torino, Italy, 19-23 September 2010.

45. Omiya, T.; Yoshida, M.; Nakazawa, M. 1 Tbit/s 256 QAM-OFDM transmission over 560 km with $14.3 \mathrm{bit} / \mathrm{s} / \mathrm{Hz}$ spectral efficiency. In Proceedings of the 18th OptoElectronics and Communications Conference held jointly with 2013 International Conference on Photonics in Switching (OECC/PS), Kyoto, Japan, 30 June-4 July 2013.

46. Nakamura, M.; Kamio, Y.; Miyazaki, T. PMD- and Dispersion-Tolerance of QPSK Homodyne Detection using a Polarization-Multiplexed Pilot Carrier. In Proceedings of the European Conference on Optical Communications, ECOC 2006, Cannes, France, 24-28 September 2006.

47. Nakamura, M.; Kamio, Y.; Miyazaki, T. Real-time 40-Gbit/s 16-QAM self-homodyne using a polarization-multiplexed pilot-carrier. In Proceedings of the Digest of the IEEE/LEOS Summer Topical Meetings, Acapulco, Mexico, 21-23 July 2008.

48. Nakamura, M.; Kamio, Y.; Miyazaki, T. Linewidth-tolerant real-time 40-Gbit/s 16-QAM self-homodyne detection using a pilot carrier and ISI suppression based on electronic digital processing. Opt. Lett. 2010, 35, 13-15.

49. Kamio, Y.; Nakamura, M.; Miyazaki, T. Pilot-Carrier Based Linewidth-Tolerant 8PSK Self-homodyne using Only One Modulator. In Proceedings of the 33rd European Conference and Ehxibition of Optical Communication (ECOC), Berlin, Germany, 16-20 September 2007.

50. Nakamura, M.; Kamio, Y.; Miyazaki, T. Linewidth-tolerant 30 Gbit/s 8-PSK self-homodyne using single modulator and phase-noise cancelling technique. Electron. Lett. 2009, 45, 368-369.

51. Qu, Z.; Fu, S.; Zhang, M; Tang, M; Shum, P.; Liu, D. Analytical Investigation on Self-Homodyne Coherent System Based on Few-Mode Fiber. IEEE Photonics Technol. Lett. 2014, 26, 74-77.

52. Puttnam, B.J.; Sakaguchi, J.; Delgado-Mendinueta, J.M.; Klaus, W.; Awaji, Y; Wada, N.; Kanno, A.; Kawanishi, T. Investigating self-homodyne coherent detection in a 19-core spatial-division-multiplexed transmission link. In Proceedings of the 38th European Conference and Exhibition on Optical Communications (ECOC), Amsterdam, The Netherlands, 16-20 September 2012.

53. ITU. Recommendation G.975 forward Error Correction for Submarine Systems. In Proceedings of the World Telecommunications Standardization Assembly, Montreal, QC, Canada, 27 September-6 October 2000.

54. Puttnam, B.J.; Delgado-Mendinueta, J.M.; Sakaguchi, J.; Luis, R.S.; Klaus, W.; Awaji, Y; Wada, N.; Kanno, A.; Kawanishi, T. 210Tb/s Self-Homodyne PDM-WDM-SDM Transmission with DFB lasers in a 19-Core Fiber. In Proceedings of the IEEE Photonics Society Summer Topical Meeting Series, Waikoloa, HI, USA, 8-10 July 2013. 
55. Maher, R.; Thomsen, B. Dynamic linewidth measurement technique using digital intradyne coherent receivers. Opt. Express 2011, 19, B313-B322.

56. Le Taillandier de Gabory, E.; Arikawa, M.; Ito, T.; Fukuchi, K. DWDM transmission of 128 Gb/s PM-16QAM signal over $1815 \mathrm{~km}$ of 7-core MCF using shared carrier reception for improving the received signal quality. In Proceedings of the 39th European Conference and Exhibition on Optical Communication (ECOC 2013), London, UK, 22-26 September 2013.

57. Amaya, N.; Irfan, M.; Zervas, G.; Nejabati, R.; Simeonidou, D.; Sakaguchi, J.; Klaus, W.; Puttnam, B.J.; Miyazawa, T.; Awaji, Y.; et al. First Fully-Elastic Multi-Granular Network with Space/Frequency/Time Switching Using Multi-Core Fibres and Programmable Optical Nodes. In Proceedings of the European Conference and Exhibition on Optical Communication, London, UK, 16-20 September 2012.

58. Amaya, N.; Irfan, M.; Zervas, G.; Nejabati, R.; Simeonidou, D.; Sakaguchi, J.; Klaus, W.; Puttnam, B.J.; Miyazawa, T.; Awaji, Y.; et al. Fully-elastic multi-granular network with space/frequency/time switching using multi-core fibres and programmable optical nodes. Opt. Express 2013, 21, 8658-8872.

59. Feuer, M.D.; Nelson, L.E.; Abedin, K.; Zhou, X.; Taunay, T.F.; Fini, J.F.; Zhu, B.; Isaac, R.; Harel, R.; Cohen, G.; et al. ROADM System for Space Division Multiplexing with Spatial Superchannels. In Proceedings of the Optical Fiber Communication Conference and Exposition and the National Fiber Optic Engineers Conference (OFC/NFOEC), Anaheim, CA, USA, 17-21 March 2013.

60. Sakaguchi, J; Klaus, W.; Puttnam, B.J.; Miyazawa, T.; Awaji, Y; Delgado-Mendinueta, J.M.; Luis, R.S.; Wada, N. SDM-WDM hybrid reconfigurable add-drop nodes for self-homodyne photonic networks. In Proceedings of the IEEE Photonics Society Summer Topical Meeting Series, Waikoloa, HI, USA, 8-10 July 2013.

61. Amaya, N.; Yan, S.; Channegowda, M.; Rofoee, B.R.; Shu, Y.; Rashidi, M.; Ou, Y.; Zervas, G.; Nejabati, R.; Simeonidou, D.; et al. First Demonstration of Software Defined Networking (SDN) over Space Division Multiplexing (SDM) Optical Networks. In Proceedings of the 39th European Conference and Exhibition on Optical Communication (ECOC 2013), London, UK, 22-26 September 2013.

62. Amaya, N.; Yan, S.; Channegowda, M.; Rofoee, B.R.; Shu, Y.; Rashidi, M.; Ou, Y.; Zervas, G.; Nejabati, R.; Simeonidou, D.; et al. Software defined networking (SDN) over space division multiplexing (SDM) optical networks: Features, benefits and experimental demonstration. Opt. Express 2014, 22, 3638-3647.

63. Zan, Z.; Lowery, A.J. Experimental demonstration of a flexible and stable semiconductor laser linewidth emulator. Opt. Express 2014, 18, 13880-13885.

(C) 2014 by the authors; licensee MDPI, Basel, Switzerland. This article is an open access article distributed under the terms and conditions of the Creative Commons Attribution license (http://creativecommons.org/licenses/by/3.0/). 